TITLE:

\title{
Wave-type threshold dynamics and the hyperbolic mean curvature flow
}

$\operatorname{AUTHOR}(\mathrm{S})$ :

Ginder, Elliott; Svadlenka, Karel

CITATION:

Ginder, Elliott ... [et al]. Wave-type threshold dynamics and the hyperbolic mean curvature flow. Japan Journal of Industrial and Applied Mathematics 2016, 33(2): 501-523

ISSUE DATE:

2016-07

URL:

http://hdl.handle.net/2433/230209

\section{RIGHT:}

This is a post-peer-review, pre-copyedit version of an article published in 'Japan Journal of Industrial and Applied Mathematics'. The final authenticated version is available online at: http://dx.doi.org/10.1007/s13160-016-0221-0.; The full-text file will be made open to the public on 1 July 2017 in accordance with publisher's 'Terms and Conditions for Self-Archiving'.; この論文は出版社版でありません。引用の際には出版社版をご確認ご利用ください。; This is not the published version. Please cite only the published version. 
Noname manuscript No.

(will be inserted by the editor)

\title{
Wave-type threshold dynamics and the hyperbolic mean curvature flow
}

\author{
Elliott Ginder • Karel Svadlenka
}

Received: date / Accepted: date

\begin{abstract}
We introduce a method for computing interfacial motions governed by curvature dependent acceleration. Our method is a thresholding algorithm of the MBO-type which, instead of utilizing a diffusion process, thresholds evolution by the wave equation to obtain the desired interfacial dynamics. We also develop the numerical method and present results of its application, including investigations of volume preserving and multiphase motions.
\end{abstract}

\section{Introduction}

In this report, we propose a numerical scheme for the computation of the so-called hyperbolic mean curvature flow (HMCF)

$$
\gamma_{t t}(t, s)=-\kappa(t, s) \nu(t, s), \quad \gamma(0, s)=\gamma_{0}(s), \quad \gamma_{t}(0, s)=v_{0}(s) \nu_{0} .
$$

Here $\gamma:[0, T) \times I \rightarrow \mathbb{R}^{2}$ is a family of smooth curves, $\kappa$ denotes the curvature, $\nu$ is the unit outer normal vector and subscripts denote partial derivatives, e.g., $\gamma_{t}=\partial \gamma / \partial t$.

This and similar types of hyperbolic problems have been derived as models of oscillatory interface motions. For example, [12] derives a model equation based on Hamilton's principle, considering stationary points of a geometrical action with local energy density consisting of kinetic and internal energy

E. Ginder

Research Institute for Electronic Science, Hokkaido University, Sapporo, Japan

E-mail: eginder@es.hokudai.ac.jp

K. Svadlenka

Department of Mathematics, Kyoto University, Kyoto, Japan

E-mail: karel@math.kyoto-u.ac.jp 
If the initial velocity is normal to the interface, the velocity remains normal during the motion, which leads to the equation

$$
\gamma_{t t}=e \kappa \nu-\nabla e
$$

Employing the variational structure, the authors show that the flow is locally well-posed and study conditions under which blow-up occurs. In the case of graphs, they show uniqueness of solution satisfying a certain entropy condition.

A similar equation

$$
\gamma_{t t}=\kappa \nu-\nabla e
$$

which is found to be related to a hyperbolic Monge-Ampère equation, is studied in $[10,11]$ with an emphasis on the development of singularities. The derivation of the equation is not given but its relation to equations for the motion of relativistic strings in Minkowski space is mentioned.

On the other hand, regarding damped oscillations, which appear on the solid-liquid interface of some crystals during melting or crystallization, $[6,15]$ derived the model equation

$$
\rho v_{t}+\beta v=\left(\psi+\psi^{\prime \prime}\right) \kappa-F
$$

(also see the references therein). Here, $v, \frac{d v}{d t}$ are the normal velocity and normal acceleration, and $\psi, \beta, \rho, F$ denote the interfacial energy, kinetic coefficient, effective density and a driving force for crystallization, respectively.

Equation (1) is also addressed in [7], where local existence and relations for the evolution of geometrical quantities are shown. It is a special case of a model equation for the motion of bubbles obtained in [8],

$$
\mu u_{t}=-p \nu-\sigma \kappa \nu+f-\mu u(\nabla \cdot u-\nu \cdot D u \cdot \nu)-u \mu_{t} .
$$

Here, $u_{t}$ is the derivative of the velocity vector, $\mu$ denotes mass density, $p$ is a factor corresponding to pressure, $\sigma$ is surface tension and $f$ denotes additional sources of momentum.

Numerical solution of the above hyperbolic mean curvature flows has been addressed only scarcely. Except for front tracking schemes, [6] develops a crystalline algorithm for the motion of closed convex polygonal curves. These methods cannot directly manage singularities, such as topological changes or the presence of junctions in the multiphase case, yet such problems can be resolved by adopting the level-set approach. A level-set method for curvature dependent accelerations based on the results of Sethian and Osher is presented in [8]. In this method, a nonlinear ill-posed problem has to be solved and it is not clear how the ideas can be extended to the multiphase setting. Just after this article was finished, we learned about the interesting work [1], where an algorithm based on the level-set approach is developed for the computation of time sections of minimal surfaces in Minkowski space, which represents a relativistic generalization of the HMCF considered here.

In this paper we aim at constructing a numerical scheme based on threshold dynamics of the MBO type. The MBO algorithm was presented in [13] and 
is based on the fact that frequent thresholding of the solution to the heat equation approximates the evolution of level sets according to the standard (parabolic) mean curvature flow. It is not obvious that such a method would be feasible for curvature accelerated motions. Nevertheless, investigating the formulae for the solution to the wave equation has revealed that level sets of the solutions whose initial condition is the signed distance to the interface, evolve with normal acceleration equal to their mean curvature, when the thresholding interval approaches zero.

A justification of the above observation can be given by the following formal analysis of radially symmetric solutions to the wave equation. The $d$ dimensional wave equation $u_{t t}=c^{2} \Delta u$ in polar coordinates reads:

$$
u_{t t}=c^{2}\left(u_{r r}+\frac{d-1}{r} u_{r}+\frac{1}{r^{2}} \Delta_{S^{d-1}} u\right),
$$

where $\Delta_{S^{d-1}}$ is the Laplace-Beltrami operator on the $(d-1)$-sphere. Assuming radial independence, this reduces to

$$
u_{t t}=c^{2}\left(u_{r r}+\frac{d-1}{r} u_{r}\right) .
$$

Integrating twice with respect to time yields

$$
u(\tau, r)=u_{0}(r)+v_{0}(r) \tau+c^{2} \int_{0}^{\tau} \int_{0}^{s}\left(u_{r r}+\frac{d-1}{r} u_{r}\right) d t d s,
$$

where $v_{0}$ denotes the initial velocity. We would like to find the relation satisfied by the zero level-set position $r_{n+1}$ (that is, $u\left(\tau, r_{n+1}\right)=0$ ), assuming that time is discretized and that the initial conditions are given by signed distance functions $d_{n}, d_{n-1}$ to the previous two interface positions $r_{n}, r_{n-1}$ at times 0 and $-\tau$, respectively. Hence, setting $u_{0}(r)=d_{n}=r_{n}-r$ and $v_{0}(r)=$ $\left(d_{n}-d_{n-1}\right) / \tau=\left(r_{n}-r_{n-1}\right) / \tau$, we derive

$$
u(\tau, r)=2 r_{n}-r_{n-1}-r+c^{2} \int_{0}^{\tau} \int_{0}^{s}\left(u_{r r}+\frac{d-1}{r} u_{r}\right) d t d s .
$$

Now, up to first order, $u_{r r}(t, r)=u_{r r}(0, r)+O(t)$ and, similarly $u_{r}(t, r)=$ $u_{r}(0, r)+O(t)$. Since the initial conditions are one-dimensional signed distance functions, we can compute their derivatives to obtain that near the interface:

$$
\begin{aligned}
u(\tau, r) & =2 r_{n}-r_{n-1}-r+c^{2} \int_{0}^{\tau} \int_{0}^{s}\left(-\frac{d-1}{r}\right) d t d s+O\left(\tau^{3}\right) \\
& =2 r_{n}-r_{n-1}-r-\frac{c^{2}(d-1) \tau^{2}}{2 r}+O\left(\tau^{3}\right) .
\end{aligned}
$$

The radius $r_{n+1}$ at the next time step is given by the zero level-set position of $u(\tau, r)$, hence

$$
\frac{r_{n+1}-2 r_{n}+r_{n-1}}{\tau^{2}}=-\frac{c^{2}(d-1)}{2 r_{n+1}}+O(\tau)
$$


Since the left-hand side represents a discretization of the acceleration, this suggests that the interface evolves approximately according to hyperbolic mean curvature flow if we take $c^{2}=\frac{2}{d-1}$. In particular, for $d=2$ we have $c^{2}=2$, and for $d=3$ we obtain $c=1$.

In the following, we will present and formally justify the resulting algorithm (which we call HMBO, the hyperbolic MBO algorithm) in the two-dimensional setting, together with its device for propagating velocities over the redistancing step without the need for explicit computation of velocities. We then comment on potential extensions of our scheme to more general flows, such as those involving phase volume constraints and the motion of junctions. We also include several numerical results and numerical confirmations regarding the proposed method.

\section{The HMBO algorithm}

The proposed HMBO algorithm for a numerical approximation $\left\{\gamma_{n}\right\}_{n=0}^{N}$ of the motion (1) in the case of a planar closed curve reads as follows, where $N$ is a positive integer.

Given: initial curve $\gamma_{0}$, its normal velocity $v_{0}$, a final time $T$ and a time step $\tau=T / N$.

1. Extend $v_{0}$ in a suitable way to a neighborhood of $\gamma_{0}$.

2. For $t \in[0, \tau]$ solve the initial value problem

$$
u_{t t}(t, x)=\Delta u(t, x), \quad u(0, x)=d_{0}(x), \quad u_{t}(0, x)=-v_{0}(x),
$$

where $d_{0}(x)$ is the signed distance function to $\gamma_{0}$.

3. Define $\gamma_{1}$ as the zero level set of $u(x, \tau)$.

4. For $n=1,2, \ldots, N-1$ repeat

(a) For $t \in[0, \tau]$ solve the initial value problem

$$
u_{t t}(t, x)=2 \Delta u(t, x), \quad u(0, x)=2 d_{n}(x)-d_{n-1}(x), \quad u_{t}(0, x)=0
$$

where $d_{n}(x)$ is the signed distance function to $\gamma_{n}$.

(b) Define $\gamma_{n+1}$ as the zero level set of $u(x, \tau)$.

In this section, we explain the ideas behind the derivation of the above $\mathrm{HMBO}$ algorithm and provide a formal justification for its convergence. We divide the explanation into two parts. We begin by addressing the construction of the first curve $\gamma_{1}$ (steps $1-3$ in the algorithm), which will clarify the reason why a threshold-type scheme for the wave equations leads to accelerations proportional to curvature. Then we treat further HMBO steps (step 4 in the algorithm), focusing on obtaining the propagation of interfacial velocities throughout the evolution. 
2.1 The first time step of the HMBO algorithm

Given an initial closed smooth planar curve $\gamma_{0}$ and its smooth initial normal velocity $v_{0}$, we construct an approximation $\gamma_{1}$ of the curve's evolution by $\mathrm{HMCF}$ at time $\tau$. This is done as follows:

1. Extend $v_{0}$ to the neighborhood of $\gamma_{0}$ in $\mathbb{R}^{2}$ as

$$
v_{0}\left(x_{1}, x_{2}\right)=v_{0}\left(\hat{x}_{1}, \hat{x}_{2}\right),
$$

where $\left(\hat{x}_{1}, \hat{x}_{2}\right)$ is the orthogonal projection of $\left(x_{1}, x_{2}\right)$ on $\gamma_{0}$, i.e., the nearest point to $\left(x_{1}, x_{2}\right)$ on the curve $\gamma_{0}$.

2. Solve the initial value problem

$$
u_{t t}=c^{2} \Delta u, \quad u(0, x)=d(x), \quad u_{t}(0, x)=-v_{0}(x),
$$

where $d(x)$ is the signed distance function to $\gamma_{0}$, and where we take $c=$ 1 here, (but we keep the general coefficient $c$ for later convenience). We remark that the velocity $v_{0}$ is defined only in a neighborhood of $\gamma_{0}$ but, since $\gamma_{0}$ is smooth and $\tau$ can be taken small enough, this fact will not hinder the subsequent step due to the finite speed of propagation.

3. Define $\gamma_{1}$ as the zero level set of $u(x, \tau)$.

We now examine the evolution of the interface, making use of the explicit representation formula. Here and in the sequel, $x=\left(x_{1}, x_{2}\right)$ and $y=\left(y_{1}, y_{2}\right)$ are points in $\mathbb{R}^{2}$. Using the Poisson formula, we find that the solution $u$ to (4) reads

$$
u(t, x)=\frac{1}{2 \pi c t} \int_{B(x, c t)} \frac{d(y)+\nabla d(y) \cdot(y-x)-t v_{0}(y)}{\sqrt{c^{2} t^{2}-|y-x|^{2}}} d y,
$$

where $B(x, r)$ denotes the ball centered at $x$ with radius $r$.

In order to analyze the resulting motion, we take a point on the interface and rotate and translate the coordinate system, so that the point becomes the origin and the outer normal to the interface points in the direction of the $x_{2}$ axis. We assume that the interface is smooth in a neighborhood of the origin. Then the value of the signed distance function at any point $y \in B(x, c t)$ can be approximated by the following Taylor expansion (see [2]), provided that $x$ in (5) is close to the origin and $t$ is sufficiently small (depending on the smoothness of the interface):

$$
d\left(y_{1}, y_{2}\right)=y_{2}+\frac{1}{2} \kappa y_{1}^{2}+\frac{1}{6} \kappa_{x_{1}} y_{1}^{3}-\frac{1}{2} \kappa^{2} y_{1}^{2} y_{2}+\sum_{|\alpha|=4} e_{\alpha}(y) y^{\alpha} .
$$

Here $\kappa$ is the curvature of the interface at the chosen point (now the origin) and the $e_{\alpha}$ 's are smooth functions. We remark that the error functions, denoted by $e_{\alpha}$ (with $\alpha$ multiindex), will vary from place to place and are always assumed to be smooth and bounded functions of their variables.

We now proceed to calculate the contribution of each term in the signed distance expansion to the solution $u(t, x)$ of the wave equation. 
For the first term $y_{2}$ we have

$$
\begin{aligned}
u^{1}(t, x) & =\frac{1}{2 \pi c t} \int_{B(x, c t)} \frac{y_{2}+\left(\begin{array}{l}
0 \\
1
\end{array}\right) \cdot\left(\begin{array}{l}
y_{1}-x_{1} \\
y_{2}-x_{2}
\end{array}\right)}{\sqrt{c^{2} t^{2}-|y-x|^{2}}} d y \\
& =\frac{1}{2 \pi c t} \int_{B(0,1)} \frac{2\left(c t z_{2}+x_{2}\right)-x_{2}}{c t \sqrt{1-|z|^{2}} c^{2} t^{2} d z} \\
& =\frac{1}{2 \pi} \int_{B(0,1)} \frac{x_{2}}{\sqrt{1-|z|^{2}}} d z \\
& =\frac{x_{2}}{2 \pi} \int_{0}^{1} \int_{0}^{2 \pi} \frac{r}{\sqrt{1-r^{2}}} d \theta d r \\
& =x_{2}
\end{aligned}
$$

Here we use the change of variables $z=(y-x) / c t$ to transform the domain of integration to a fixed ball $B(0,1)$. Also we use the fact that the function $z_{2} / \sqrt{1-|z|^{2}}$ is odd with respect to the $x_{2}$-axis, hence its integral over $B(0,1)$ vanishes.

By a similar calculation, the second term $\frac{1}{2} \kappa y_{1}^{2}$ gives

$$
\begin{aligned}
u^{2}(t, x) & =\frac{1}{2 \pi c t} \frac{\kappa}{2} \int_{B(x, c t)} \frac{y_{1}^{2}+\left(\begin{array}{c}
2 y_{1} \\
0
\end{array}\right) \cdot\left(\begin{array}{l}
y_{1}-x_{1} \\
y_{2}-x_{2}
\end{array}\right)}{\sqrt{c^{2} t^{2}-|y-x|^{2}}} d y \\
& =\frac{\kappa}{4 \pi} \int_{B(0,1)} \frac{3 c^{2} t^{2} z_{1}^{2}+x_{1}^{2}}{\sqrt{1-|z|^{2}}} d z \\
& =\frac{\kappa}{2}\left(c^{2} t^{2}+x_{1}^{2}\right) .
\end{aligned}
$$

The third term $\frac{1}{6} \kappa_{x_{1}} y_{1}^{3}$ contributes to the solution as follows:

$$
\begin{aligned}
u^{3}(t, x) & =\frac{\kappa_{x_{1}}}{12 \pi c t} \int_{B(x, c t)} \frac{y_{1}^{3}+\left(\begin{array}{c}
3 y_{1}^{2} \\
0
\end{array}\right) \cdot\left(\begin{array}{l}
y_{1}-x_{1} \\
y_{2}-x_{2}
\end{array}\right)}{\sqrt{c^{2} t^{2}-|y-x|^{2}}} d y \\
& =\frac{\kappa_{x_{1}}}{12 \pi} \int_{B(0,1)} \frac{9 c^{2} t^{2} x_{1} z_{1}^{2}+x_{1}^{3}}{\sqrt{1-|z|^{2}}} d z \\
& =\frac{\kappa_{x_{1}} x_{1}}{6}\left(3 c^{2} t^{2}+x_{1}^{2}\right) .
\end{aligned}
$$

The fourth term $-\frac{1}{2} \kappa^{2} y_{1}^{2} y_{2}$ yields

$$
\begin{aligned}
u^{4}(t, x) & =-\frac{\kappa^{2}}{4 \pi c t} \int_{B(x, c t)} \frac{y_{1}^{2} y_{2}+\left(\begin{array}{c}
2 y_{1} y_{2} \\
y_{1}^{2}
\end{array}\right) \cdot\left(\begin{array}{l}
y_{1}-x_{1} \\
y_{2}-x_{2}
\end{array}\right)}{\sqrt{c^{2} t^{2}-|y-x|^{2}}} d y \\
& =-\frac{\kappa^{2}}{4 \pi} \int_{B(0,1)} \frac{3 c^{2} t^{2} x_{2} z_{1}^{2}+6 c^{2} t^{2} x_{1} z_{1} z_{2}+x_{1}^{2} x_{2}}{\sqrt{1-|z|^{2}}} d z \\
& =-\frac{\kappa^{2} x_{2}}{2}\left(c^{2} t^{2}+x_{1}^{2}\right) .
\end{aligned}
$$


The velocity term is approximated by its Taylor series as follows:

$$
\begin{aligned}
u^{v}(t, x) & =\frac{1}{2 \pi c t} \int_{B(x, c t)} \frac{t v_{0}(y)}{\sqrt{c^{2} t^{2}-|y-x|^{2}}} d y \\
& =\frac{t}{2 \pi} \int_{B(0,1)} \frac{v_{0}(x+c t z)}{\sqrt{1-|z|^{2}}} d z \\
& =\frac{t}{2 \pi} \int_{B(0,1)} \frac{v_{0}(0)+\nabla v_{0}(0) \cdot(x+c t z)+\sum_{|\alpha|=2} e_{\alpha}(t, x, z)(t, x)^{\alpha}}{\sqrt{1-|z|^{2}}} d z \\
& =v_{0}(0) t+\frac{\partial}{\partial x_{1}} v_{0}(0) t x_{1}+\sum_{|\alpha|=3} e_{\alpha}(t, x)(t, x)^{\alpha},
\end{aligned}
$$

since $\frac{\partial v_{0}}{\partial x_{x}}(0)=0$.

Finally, the error term in the signed distance expansion can be evaluated as

$$
\begin{aligned}
& u^{e}(t, x) \\
& =\frac{1}{2 \pi c t} \sum_{|\alpha|=4} \int_{B(x, c t)} \frac{e_{\alpha}(y) y^{\alpha}+\nabla\left(e_{\alpha}(y) y^{\alpha}\right) \cdot(y-x)}{\sqrt{c^{2} t^{2}-|y-x|^{2}}} d y \\
& =\frac{1}{2 \pi} \sum_{|\alpha|=4} \int_{B(0,1)} \frac{e_{\alpha}(x+c t z)(x+c t z)^{\alpha}+\left.\nabla\left(e_{\alpha}(y) y^{\alpha}\right)\right|_{y=x+c t z} \cdot c t z}{\sqrt{1-|z|^{2}}} d z \\
& =\sum_{|\alpha|=4} e_{\alpha}(t, x)(t, x)^{\alpha} .
\end{aligned}
$$

The solution to the wave equation with initial condition $d$ in the neighborhood of the origin can thus be written in the following way:

$$
\begin{aligned}
u(t, x)= & x_{2}+\frac{\kappa}{2}\left(c^{2} t^{2}+x_{1}^{2}\right)+\frac{\kappa_{x_{1}} x_{1}}{6}\left(3 c^{2} t^{2}+x_{1}^{2}\right)-\frac{\kappa^{2} x_{2}}{2}\left(c^{2} t^{2}+x_{1}^{2}\right) \\
& -v_{0}(0) t-\frac{\partial v_{0}}{\partial x_{1}}(0) t x_{1}+\sum_{|\alpha|=3} e_{\alpha}(t, x)(t, x)^{\alpha} .
\end{aligned}
$$

From this result we can make the following observation. If the distance travelled by the interface in the normal direction (i.e., the $x_{2}$-direction) after time $\tau$ is denoted by $\delta_{0}$, then this distance can be calculated from the relation $u\left(\tau, 0, \delta_{0}\right)=0$. In particular,

$$
0=u\left(\tau, 0, \delta_{0}\right)=\delta_{0}+\frac{1}{2} \kappa c^{2} \tau^{2}-\frac{1}{2} \delta_{0} \kappa^{2} c^{2} \tau^{2}-v_{0}(0) \tau+\sum_{|\alpha|=3} e_{\alpha}\left(\tau, \delta_{0}\right)\left(\tau, \delta_{0}\right)^{\alpha} .
$$

This relation implies that, in terms of the order in $\tau$, the second order approximation of $\delta_{0}$ is $v_{0}(0) \tau-\frac{1}{2} \kappa c^{2} \tau^{2}$. Therefore, the error term can be estimated by $O\left(\tau^{3}\right)$ and solving the above equation for $\delta_{0}$, we obtain

$$
\delta_{0}=\frac{v_{0}(0) \tau-\frac{1}{2} \kappa c^{2} \tau^{2}+O\left(\tau^{3}\right)}{1-\frac{1}{2} \kappa^{2} c^{2} \tau^{2}}=v_{0}(0) \tau-\frac{1}{2} \kappa c^{2} \tau^{2}+O\left(\tau^{3}\right) .
$$


This means that the interface moves with initial velocity $v_{0}$ and with an acceleration equal to $-c^{2} \kappa$, the $c^{2}$-multiple of the curvature. Thus taking $c=1$, we obtain the desired approximation of the interface $\gamma_{1} \approx \gamma(\tau)$.

Remark. Regarding the problem in three dimensions, assuming for simplicity $c=1, v_{0} \equiv 0$, the signed distance can be expanded as

$$
d(y)=y_{3}+\frac{1}{2} \kappa_{1} y_{1}^{2}+\frac{1}{2} \kappa_{2} y_{2}^{2}+\text { H.O.T. },
$$

where $\kappa_{1}$ and $\kappa_{2}$ are the principal curvatures, and a similar approach employing the Kirchhoff's formula suggests the validity of our method:

$$
\begin{aligned}
u(t, x) & =\frac{1}{4 \pi t^{2}} \int_{\partial B(x, t)}\left(d(y)+\nabla d(y) \cdot(y-x)-t v_{0}(y)\right) d S(y) \\
& =x_{3}+\frac{1}{2} \kappa_{1}\left(t^{2}+x_{1}^{2}\right)+\frac{1}{2} \kappa_{2}\left(t^{2}+x_{2}^{2}\right)+\text { H.O.T. }
\end{aligned}
$$

This yields the distance in the $x_{3}$-direction traveled by interface in time $\tau$ as

$$
\delta_{0}=-\frac{1}{2}\left(\kappa_{1}+\kappa_{2}\right) \tau^{2}+O\left(\tau^{3}\right),
$$

confirming that our scheme can also be used with the three-dimensional wave equation, and likely in any higher dimension.

\subsection{Further time steps of HMBO}

If we want to apply the above scheme to all time steps, the velocities along the interface would need to be computed. This would severely complicate the numerical solution, even if the velocity field was known. The difficulties inherent to such an approach are made clear, for example, when considering evolutions that involve topological changes (e.g., interfaces that split apart, or contact each other). On the other hand, if we reset the initial velocity to zero, the interface will always accelerate from zero velocity and the accumulated velocities will not propagate. Hence, we need to provide a modification of the above scheme which inherits the velocity from the previous time step and accelerates the interface depending on its curvature.

In order to design a scheme that avoids computation of interface velocities, it is necessary to account for the position of the interface at both the previous and the current time step. Thus, it is natural to consider a vanishing initial velocity in the wave equation and an initial condition of the form

$$
u(0, x)=a d_{n-1}(x)+b d_{n}(x) .
$$

Here $d_{n}$ denotes the signed distance function to the present interface $\gamma_{n}, d_{n-1}$ is the signed distance function to the interface at previous time step $\gamma_{n-1}$, and both $a$ and $b$ denote real numbers (see Fig. 1 below). 


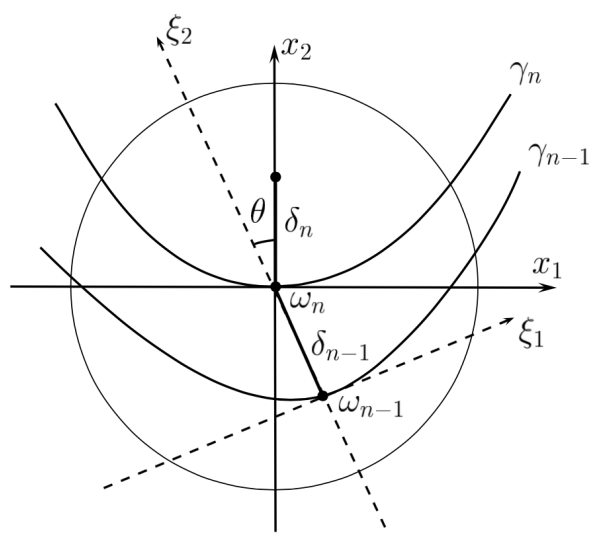

Fig. 1 The $\xi$-coordinate system.

In order to find a precise expansion of the signed distance function $d_{n-1}$, it is necessary to calculate the change in the direction of the outer normal to the interface. Let us denote the corresponding point on the previous interface $\gamma_{n-1}$ by $\omega_{n-1}$ and the curvature at that point by $\kappa_{n-1}$. Analogous notation is also used for the next interface $\gamma_{n}$. We should start from $n=1$ but we consider general $n \in \mathbb{N}$ since the coefficients $a$ and $b$ will turn out to be independent of $n$. According to (6), the solution of the wave equation (4) with initial condition $d_{n-1}$ in the $\xi$-coordinate system of Fig. 1 reads

$$
\begin{aligned}
u_{n-1}(t, \xi)= & \xi_{2}+\frac{\kappa_{n-1}}{2}\left(c^{2} t^{2}+\xi_{1}^{2}\right)+\frac{\left(\kappa_{n-1}\right)_{x_{1}} \xi_{1}}{6}\left(3 c^{2} t^{2}+\xi_{1}^{2}\right) \\
& -\frac{\kappa_{n-1}^{2} \xi_{2}}{2}\left(c^{2} t^{2}+\xi_{1}^{2}\right)-v_{0}(0) t-v_{0_{x_{1}}}(0) t \xi_{1}+\sum_{|\alpha|=3} e_{\alpha}(t, \xi)(t, \xi)^{\alpha}
\end{aligned}
$$

We want to compute the unit outer normal $\nu_{n}$ to the level set of $u_{n-1}$ at the point $\omega_{n}=(0, \delta)\left(\right.$ with $\left.\delta=\delta_{n-1}\right)$ in the $\xi$-coordinate system. Hence,

where

$$
\nu_{n}=\frac{\nabla u_{n-1}(\tau, 0, \delta)}{\left|\nabla u_{n-1}(\tau, 0, \delta)\right|}
$$

$$
\begin{aligned}
\nabla u_{n-1}(\tau, \xi)= & \left(\begin{array}{c}
\kappa_{n-1} \xi_{1}+\frac{\left(\kappa_{n-1}\right)_{x_{1}}}{2}\left(c^{2} \tau^{2}+\xi_{1}^{2}\right)-\kappa_{n-1}^{2} \xi_{1} \xi_{2}-v_{0_{x_{1}}}(0) t \\
1-\frac{\kappa_{n-1}^{2}}{2}\left(c^{2} \tau^{2}+\xi_{1}^{2}\right)
\end{array}\right) \\
& +\nabla_{\xi} \sum_{|\alpha|=3} e_{\alpha}(t, \xi)(t, \xi)^{\alpha} .
\end{aligned}
$$

This yields

$$
\nabla u_{n-1}(\tau, 0, \delta)=\left(\begin{array}{c}
\frac{\left(\kappa_{n-1}\right)_{x_{1}}}{2} c^{2} \tau^{2}-v_{0_{x_{1}}}(0) \tau \\
1-\frac{\kappa_{n-1}^{2}}{2} c^{2} \tau^{2}
\end{array}\right)+\left(\begin{array}{c}
\sum_{|\alpha|=2} e_{\alpha}(\tau, \delta)(\tau, \delta)^{\alpha} \\
\sum_{|\alpha|=2} e_{\alpha}(\tau, \delta)(\tau, \delta)^{\alpha}
\end{array}\right)
$$


and

$$
\nu_{n}=\frac{1}{\sqrt{1+\left(\nu_{n}^{1}\right)^{2}}}\left(\begin{array}{c}
\nu_{n}^{1} \\
1
\end{array}\right), \quad \text { where } \nu_{n}^{1}=-v_{0_{x_{1}}}(0) \tau+\sum_{|\alpha|=2} e_{\alpha}(\tau, \delta)(\tau, \delta)^{\alpha}
$$

From here we can calculate the deviation angle of the normal in one time step,

$$
\cos \theta=\left(\begin{array}{l}
0 \\
1
\end{array}\right) \cdot \nu_{n}=1+O\left(\tau^{2}\right)
$$

since $\delta=O(\tau)$. Hence, we conclude that $\theta=O(\tau)$.

The $x$-coordinate and $\xi$-coordinate are rotated by the vector $\nu_{n}$ and thus,

$$
\begin{aligned}
& \xi_{1}=\frac{1}{\sqrt{1+\left(\nu_{n}^{1}\right)^{2}}}\left(x_{1}+\nu_{n}^{1} x_{2}\right) \\
& \xi_{2}=\frac{1}{\sqrt{1+\left(\nu_{n}^{1}\right)^{2}}}\left(-\nu_{n}^{1} x_{1}+x_{2}\right)+\delta_{n-1} .
\end{aligned}
$$

We can now write the signed distance $d_{n-1}$ in terms of $x$, as follows (for simplicity we write $\nu$, instead of $\nu_{n}^{1}$ ):

$$
\begin{aligned}
d_{n-1}(x)= & \xi_{2}+\frac{1}{2} \kappa_{n-1} \xi_{1}^{2}+\frac{1}{6}\left(\kappa_{n-1}\right)_{x_{1}} \xi_{1}^{3}-\frac{1}{2} \kappa_{n-1}^{2} \xi_{1}^{2} \xi_{2}+\sum_{|\alpha|=4} e_{\alpha}(\xi) \xi^{\alpha} \\
= & \frac{-\nu x_{1}+x_{2}}{\sqrt{1+\nu^{2}}}+\delta_{n-1}+\frac{\kappa_{n-1}}{2\left(1+\nu^{2}\right)}\left(x_{1}+\nu x_{2}\right)^{2}+\frac{\left(\kappa_{n-1}\right)_{1}}{6 \sqrt{1+\nu^{2}}}\left(x_{1}+\nu x_{2}\right)^{3} \\
& -\frac{\kappa_{n-1}^{2}\left(x_{1}+\nu x_{2}\right)^{2}}{2\left(1+\nu^{2}\right)}\left(\frac{-\nu x_{1}+x_{2}}{\sqrt{1+\nu^{2}}}+\delta_{n-1}\right)+\sum_{|\alpha|=4} e_{\alpha}\left(x, \delta_{n-1}\right)\left(x, \delta_{n-1}\right)^{\alpha} \\
= & x_{2}+\delta_{n-1}+\frac{1}{2} \kappa_{n-1} x_{1}^{2}+\frac{1}{6}\left(\kappa_{n-1}\right)_{x_{1}} x_{1}^{3}-\frac{1}{2} \kappa_{n-1}^{2} x_{1}^{2} x_{2}-\nu x_{1}-\frac{\nu^{2}}{2} x_{2} \\
& +\frac{\kappa_{n-1}}{2}\left(-\nu^{2} x_{1}^{2}+2 \nu x_{1} x_{2}+\nu^{2} x_{2}^{2}\right)+\frac{\left(\kappa_{n-1}\right)_{x_{1}}}{6}\left(3 \nu x_{1}^{2} x_{2}+3 \nu^{2} x_{1} x_{2}^{2}\right) \\
& -\frac{\left(\kappa_{n-1}\right)_{x_{1}}}{4} \nu^{2} x_{1}^{3}-\frac{\kappa_{n-1}^{2}}{2}\left(-\nu x_{1}^{3}-2 \nu^{2} x_{1}^{2} x_{2}+2 \nu x_{1} x_{2}^{2}+\nu^{2} x_{2}^{3}\right) \\
& +\frac{3 \kappa_{n-1}^{2}}{4} \nu^{2} x_{1}^{2} x_{2}-\frac{\kappa_{n-1}^{2}}{2\left(1+\nu^{2}\right)}\left(x_{1}+\nu x_{2}\right)^{2} \delta_{n-1} \\
& +\sum_{|\alpha|=4} e_{\alpha}\left(x, \delta_{n-1}\right)\left(x, \delta_{n-1}\right)^{\alpha}+\sum_{i \geq 3} e_{i}(x) \nu^{i} .
\end{aligned}
$$

Because of (9), the last error term can be written as $\sum_{|\alpha|=3} e_{\alpha}\left(\tau, \delta_{n-1}, x\right)\left(\tau, \delta_{n-1}\right)^{\alpha}$. 
Computing the solution of the wave equation with initial condition $d_{n-1}$ and zero initial velocity, we obtain

$$
\begin{aligned}
u_{n-1}(t, x)= & x_{2}+\delta_{n-1}+\frac{\kappa_{n-1}}{2}\left(c^{2} t^{2}+x_{1}^{2}\right)+\frac{\left(\kappa_{n-1}\right)_{x_{1}} x_{1}}{6}\left(3 c^{2} t^{2}+x_{1}^{2}\right) \\
& -\frac{\kappa_{n-1}^{2} x_{2}}{2}\left(c^{2} t^{2}+x_{1}^{2}\right)-\nu x_{1}-\frac{\nu^{2}}{2} x_{2}+\frac{\kappa_{n-1}}{2}\left[-\nu^{2}\left(c^{2} t^{2}+x_{1}^{2}\right)\right. \\
& \left.+2 \nu x_{1} x_{2}+\nu^{2}\left(c^{2} t^{2}+x_{2}^{2}\right)\right]+\frac{\left(\kappa_{n-1}\right)_{x_{1}}}{6}\left[3 \nu x_{2}\left(c^{2} t^{2}+x_{1}^{2}\right)\right. \\
& \left.+3 \nu^{2} x_{1}\left(c^{2} t^{2}+x_{2}^{2}\right)\right]-\frac{\left(\kappa_{n-1}\right)_{x_{1}}}{4} \nu^{2} x_{1}\left(3 c^{2} t^{2}+x_{1}^{2}\right) \\
& +\frac{\kappa_{n-1}^{2}}{2}\left[\nu x_{1}\left(c^{2} t^{2}+x_{1}^{2}-2 x_{2}^{2}\right)+\frac{1}{2} \nu^{2} x_{2}\left(c^{2} t^{2}+7 x_{1}^{2}-x_{2}^{2}\right)\right] \\
& -\frac{\kappa_{n-1}^{2} \delta_{n-1}}{2\left(1+\nu^{2}\right)}\left[c^{2} t^{2}+x_{1}^{2}+2 \nu x_{1} x_{2}+\nu^{2}\left(c^{2} t^{2}+x_{2}^{2}\right)\right] \\
& +\sum_{|\alpha|=4} e_{\alpha}(t, x)(t, x)^{\alpha}+\sum_{|\alpha|=3} e_{\alpha}\left(\tau, \delta_{n-1}, t, x\right)\left(\tau, \delta_{n-1}\right)^{\alpha},
\end{aligned}
$$

where we have used the calculations of the integrals from Poisson formula in Section 2.1. The solution for for the initial condition $a d_{n-1}+b d_{n}$ evaluated at the point $(t, x)=\left(\tau, 0, \delta_{n}\right)$ thus reads

$$
\begin{aligned}
& u_{n}\left(\tau, 0, \delta_{n}\right)= \\
& (a+b) \delta_{n}+a \delta_{n-1}+\frac{a \kappa_{n-1}+b \kappa_{n}}{2} c^{2} \tau^{2}-\frac{a \kappa_{n-1}^{2}+b \kappa_{n}^{2}}{2} \delta_{n} c^{2} \tau^{2} \\
& +a\left\{-\frac{\nu^{2}}{2} \delta_{n}+\frac{\kappa_{n-1}}{2} \nu^{2} \delta_{n}^{2}+\frac{\left(\kappa_{n-1}\right)_{x_{1}}}{2} \nu \delta_{n} c^{2} \tau^{2}\right. \\
& \left.-\frac{\kappa_{n-1}^{2}}{2}\left[-\frac{1}{2} \nu^{2} \delta_{n} c^{2} \tau^{2}+\nu^{2} \delta_{n}^{3}\right]-\frac{\kappa_{n-1}^{2}}{2\left(1+\nu^{2}\right)} \delta_{n-1}\left[c^{2} \tau^{2}\left(1+\nu^{2}\right)+\nu^{2} \delta_{n}^{2}\right]\right\} \\
& +\sum_{|\alpha|=4} e_{\alpha}\left(\tau, \delta_{n}\right)\left(\tau, \delta_{n}\right)^{\alpha}+\sum_{|\alpha|=3} e_{\alpha}\left(\tau, \delta_{n-1}, \delta_{n}\right)\left(\tau, \delta_{n-1}\right)^{\alpha} \text {. }
\end{aligned}
$$

The distance $\delta_{n}$ traveled by the interface in the normal direction then satisfies $u_{n}\left(t, 0, \delta_{n}\right)=0$, which can be written as

$$
\begin{aligned}
0= & -\delta_{n}^{3} a \nu^{2} \frac{\kappa_{n-1}^{2}}{2}+\delta_{n}^{2} a \nu^{2} \frac{\kappa_{n-1}^{2}}{2}\left(1-\frac{\delta_{n-1}}{1+\nu^{2}}\right) \\
& +\delta_{n}\left(a+b-\frac{a \kappa_{n-1}^{2}+b \kappa_{n}^{2}}{2} c^{2} \tau^{2}-\frac{a}{2} \nu^{2}+\frac{a}{2} \nu c^{2} \tau^{2}\left(\left(\kappa_{n-1}\right)_{x_{1}}+\frac{1}{2} \nu \kappa_{n-1}^{2}\right)\right) \\
& +a \delta_{n-1}+\frac{a \kappa_{n-1}+b \kappa_{n}}{2} c^{2} \tau^{2}-\frac{\kappa_{n-1}^{2}}{2} a \delta_{n-1} c^{2} \tau^{2}+\text { error terms, }
\end{aligned}
$$

where the error terms are of order $O\left(\tau^{3}+\delta_{n-1}^{3}+\delta_{n}^{4}\right)$. Moreover, the quantities $\delta_{n-1}$ and $\nu$ are of order $O(\tau)$, at worst. Since the lowest order approximation of the above equation is

$$
\delta_{n}(a+b)+a \delta_{n-1}+\frac{a \kappa_{n-1}+b \kappa_{n}}{2} c^{2} \tau^{2}=0,
$$

we see that $\delta_{n}$ must be of order $O\left(\delta_{n-1}+\tau^{2}\right)$, which is at most $O(\tau)$. Hence the $\delta_{n}^{2}$ and $\delta_{n}^{3}$ terms (including their coefficients) are of order $O\left(\tau^{4}\right)$ or higher 
and can be neglected. In this way we obtain the solution $\delta_{n}$ to the equation as

$$
\begin{aligned}
\delta_{n} & =-\frac{a \delta_{n-1}+\frac{a \kappa_{n-1}+b \kappa_{n}}{2} c^{2} \tau^{2}+O\left(\tau^{3}+\delta_{n-1}^{3}\right)}{a+b-\frac{a \kappa_{n-1}^{2}+b \kappa_{n}^{2}}{2} c^{2} \tau^{2}-\frac{a}{2} \nu^{2}} \\
& =\frac{-a}{a+b} \delta_{n-1}-\frac{a \kappa_{n-1}+b \kappa_{n}}{2(a+b)} c^{2} \tau^{2}+O\left(\tau^{3}\right) .
\end{aligned}
$$

It is natural to choose $a, b$ so that $-\frac{a}{a+b}=1$ and $a+b=1$, i.e., $a=-1, b=2$. Moreover, setting $c^{2}=2$ yields

$$
\delta_{n}=\delta_{n-1}-\left(2 \kappa_{n}-\kappa_{n-1}\right) \tau^{2}+O\left(\tau^{3}\right),
$$

which is the desired relation between $\delta_{n}$ and $\delta_{n-1}$.

By (9), the direction of the outer normal changes with order $\tau$, and so one can see that the above is a correct approximation by considering the onedimensional point-mass motion with initial velocity $v_{0}$ and acceleration $-\kappa(t)$ (thus solving $\left.x^{\prime \prime}(t)=-\kappa(t), x(0)=0, x^{\prime}(0)=v_{0}\right)$. The solution is

$$
x(t)=v_{0} t-\int_{0}^{t} \int_{0}^{s} \kappa(u) d u d s .
$$

Our algorithm (10), including the initial step from the previous subsection, gives the approximation

$$
\begin{aligned}
\delta_{0} & =v_{0} \tau-\frac{1}{2} \kappa_{0} \tau^{2}+O\left(\tau^{3}\right), \\
\delta_{1} & =v_{0} \tau+\frac{1}{2} \kappa_{0} \tau^{2}-2 \kappa_{1} \tau^{2}+O\left(2 \tau^{3}\right), \\
& \vdots \\
\delta_{k} & =v_{0} \tau-\frac{1}{2} \kappa_{0} \tau^{2}-\tau^{2} \sum_{i=1}^{k} \kappa_{i}+\left(\kappa_{0}-\kappa_{k}\right) \tau^{2}+O\left((k-1) \tau^{3}\right) .
\end{aligned}
$$

The corresponding total distance is

$$
\tilde{x}(k \tau)=\sum_{j=0}^{k-1} \delta_{j}=k v_{0} \tau-k \frac{1}{2} \kappa_{0} \tau^{2}-\tau^{2} \sum_{j=0}^{k-1} \sum_{i=1}^{k-j} \kappa_{j}+O(\tau),
$$

which converges as $\tau \rightarrow 0$ to the function (11) by the trapezoidal rule. The above analysis also shows that the accumulation of errors through time does not spoil the approximation of the interface, at least until the development of singularities. 


\section{Numerical Tests and Properties}

Thresholding dynamical algorithms have the computational advantage that there is no need to calculate curvatures, and singularities are implicitly handled by the partial differential equation. Moreover, they are extremely simple to implement (here, we need only solve the wave equation). Thus, one can construct the corresponding numerical method in a number of ways-we will make use of standard finite differences, as well as minimizing movements for use in investigating volume preserving motions.

Since we are considering an interfacial motion embedded in the solution to a hyperbolic equation, care needs to be taken when detecting the interface and constructing the signed distance functions. In particular, the precise location of the interface is needed. Without this information, errors arising from its approximation will propagate via the evolution of the signed distance functions.

In the same vein, care must be taken in the numerical implementation of the initial conditions, as the piecewise linear interpolation can introduce initial interfaces that are not smooth. One approach to alleviating issues caused by this is to use one step of the original (parabolic) MBO algorithm to smooth the initial interface.

We will first perform a convergence test for an idealized version of our algorithm. This test assumes that no errors are introduced under spacial discretization. Our second test introduces the spacial discretization, but computes signed distance functions utilizing an idealized representation of the interface. The final test removes this idealization and examines our algorithm's order of convergence using standard finite differences.

\subsection{Idealized numerical convergence}

This section investigates the convergence rate of our algorithm for a simple test problem, under a slight idealization. For a circle evolving by (1) with initial normal velocity $v_{0}$ (we assume $v_{0} \leq 0$ for simplicity), one can compute the evolution of the circle's radius $r(t)$ analytically by solving

$$
r^{\prime \prime}(t)=-\frac{1}{r(t)}, \quad r(0)=r_{0}, \quad r^{\prime}(0)=v_{0} .
$$

Multiplying the equation by $r^{\prime}(t)$ and integrating we obtain

$$
r^{\prime}(t)=-\sqrt{-2 \log r+C_{1}},
$$

where $C_{1}=2 \log r_{0}+v_{0}^{2}$ is determined by the initial conditions. Integrating,

$$
e^{C_{1} / 2} \sqrt{\frac{\pi}{2}} \operatorname{erf}\left(\sqrt{\frac{C_{1}}{2}-\log r(t)}\right)=t+C_{2}, \quad \text { where } C_{2}=r_{0} \sqrt{\frac{\pi}{2}} e^{\frac{v_{0}^{2}}{2}} \operatorname{erf}\left(\frac{v_{0}}{\sqrt{2}}\right) .
$$

In particular, $C_{2}=0$ for zero intial velocity. 
The solution can thus be expressed as

$$
r(t)=\exp \left(\frac{C_{1}}{2}-\left[\operatorname{erf}^{-1}\left(\sqrt{\frac{2}{\pi}} e^{-C_{1} / 2}\left(t+C_{2}\right)\right)\right]^{2}\right),
$$

which yields a more simple form for zero initial velocity:

$$
r(t)=r_{0} e^{-\left[\operatorname{erf}^{-1}\left(\sqrt{\frac{2}{\pi}} \frac{t}{r_{0}}\right)\right]^{2}} .
$$

Since the function $\operatorname{erf}^{-1}(x)$ tends to infinity when $x \rightarrow 1-$, the radius vanishes in a finite time, which can be computed from the relation $\sqrt{2 / \pi} e^{-C_{1} / 2}\left(t_{e}+\right.$ $\left.C_{2}\right)=1$. After substituting values of integration constants, this gives

$$
t_{e}=r_{0} \sqrt{\frac{\pi}{2}} e^{v_{0}^{2} / 2}\left(1+\operatorname{erf}\left(\frac{v_{0}}{\sqrt{2}}\right)\right) .
$$

We remark that the extinction time for zero initial velocity is $t_{e}=r_{0} \sqrt{\pi / 2}$ (see Fig. 2).

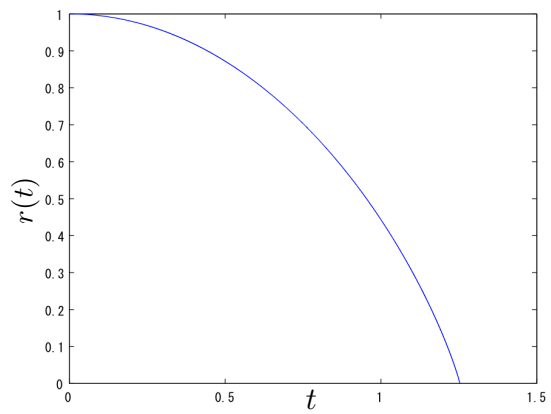

Fig. 2 Graph of the solution for $r_{0}=1, v_{0}=0$.

Let us now calculate the numerical approximation due to our scheme. That is, we consider two circles of radii $r_{0}>r_{1}$, centered at the origin, which describe the initial conditions. We then compute the radius of the circle given by the 0 -level set of the solution (after time $t$ ) to the problem

$$
u_{t t}=c^{2} \Delta u, \quad u(0, x)=2 d_{1}(x)-d_{0}(x), \quad u_{t}(0, x)=0 .
$$

Here, $c^{2}=2$ and $d_{i}$ is the signed distance function to the circle of radius $r_{i}$, which can be written as $d_{i}(x)=r_{i}-|x|$. Hence, denoting $r=2 r_{1}-r_{0}$, the initial condition is given by $u(0, x)=r-|x|$ and the solution of the above initial value problem (sufficiently far away from the origin) is

$$
\begin{aligned}
u(t, x) & =\frac{1}{2 c \pi t} \int_{B(x, c t)} \frac{r-|y|-\frac{y}{|y|} \cdot(y-x)}{\sqrt{c^{2} t^{2}-|y-x|^{2}}} d y \\
& =\frac{1}{2 \pi} \int_{B(0,1)} \frac{r-2|x+c t z|+\frac{|x|^{2}+c t x \cdot z}{|x+c t z|}}{\sqrt{1-|z|^{2}}} d z .
\end{aligned}
$$


Since the initial condition is radially symmetric and there is no interaction with a boundary, we can restrict the values of $x$ to points on the $x$-axis of the form $x=(\xi, 0)$, where $\xi>0$. Then the above simplifies to

$$
u(t, \xi, 0)=r-\frac{1}{2 \pi} \int_{0}^{1} \int_{0}^{2 \pi} \frac{\xi^{2}+3 c t \rho \xi \cos \theta+2 c^{2} t^{2} \rho^{2}}{\sqrt{1-\rho^{2}} \sqrt{\xi^{2}+2 c t \rho \xi \cos \theta+c^{2} t^{2} \rho^{2}}} \rho d \theta d \rho .
$$

The exact form of the solution to the above equation is difficult to find, since it involves elliptic integrals. Therefore, let us instead assume that $t=\tau$ is small and expand the integrand as a Taylor series in time. To this end, let us denote

$$
G(t)=\frac{\xi^{2}+3 c t \rho \cos \theta+2 c^{2} t^{2} \rho^{2}}{\sqrt{\xi^{2}+2 c t \rho \xi \cos \theta+c^{2} t^{2} \rho^{2}}},
$$

and calculate the first and second derivatives of $G$ at $t=0$ :

$$
G(0)=\xi, \quad G^{\prime}(0)=2 c \rho \cos \theta, \quad G^{\prime \prime}(0)=\frac{3 c^{2}}{\xi} \rho^{2} \sin ^{2} \theta .
$$

We can thus approximate the solution at points $(\xi, 0)$ as

$$
\begin{aligned}
& u(\tau, \xi, 0) \\
& =r-\frac{1}{2 \pi} \int_{0}^{1} \int_{0}^{2 \pi} \frac{\rho}{\sqrt{1-\rho^{2}}}\left(\xi+2 c \tau \rho \cos \theta+\frac{3 c^{2}}{2 \xi} \tau^{2} \rho^{2} \sin ^{2} \theta+O\left(\tau^{3}\right)\right) d \theta d \rho \\
& =r-\xi-\frac{c^{2}}{2 \xi} \tau^{2}+O\left(\tau^{3}\right) .
\end{aligned}
$$

To determine the position $\xi$ of the interface after time $\tau$, we need to solve

$$
r-\xi-\frac{c^{2}}{2 \xi} \tau^{2}=O\left(\tau^{3}\right)
$$

The corresponding quadratic equation $\xi^{2}-\left(r+O\left(\tau^{3}\right)\right) \xi+\frac{1}{2} c^{2} \tau^{2}=0$ has two roots but since for vanishing times we want to recover the value $\xi=r$, we take the corresponding root

$$
\xi=\frac{1}{2}\left(r+\sqrt{r^{2}-2 c^{2} \tau^{2}}\right)+O\left(\tau^{3}\right)=r-\frac{1}{r} \tau^{2}+O\left(\tau^{3}\right) .
$$

Combining this result with our considerations regarding the first HMBO step, we obtain the following HMBO algorithm for the special case of a circle.

1. Prescribe initial radius $r_{0}$, initial velocity $v_{0}$ and time step $\tau=t_{e} / N$, where $t_{e}$ is the extinction time (14) and $N$ is a positive integer.

2. Set

$$
r_{1}=r_{0}-\frac{\tau^{2}}{2 r_{0}} .
$$

3. Repeat the following for $n=2, \ldots, N$ :

$$
r_{n}=2 r_{n-1}-r_{n-2}-\frac{\tau^{2}}{2 r_{n-1}-r_{n-2}}
$$


We test the resulting scheme for $r_{0}=1$ and $v_{0}=0$. The results are shown in the Table 1, where the error is taken at the half time to extinction, i.e.,

$$
\text { error }=\left|r_{\lfloor N / 2\rfloor}-r\left(t_{e} / 2\right)\right|=\left|r_{\lfloor N / 2\rfloor}-\exp \left(-\left[\operatorname{erf}^{-1}\left(\frac{1}{2}\right)\right]^{2}\right)\right| .
$$

We observe exactly linear convergence with respect to the time step $\tau$ as predicted by (12), which shows that the proposed HMBO algorithm gives correct (i.e., convergent) result when the initial curve is a circle.

\begin{tabular}{|c|c|c|}
\hline division number $N$ & error at time $t_{e} / 2$ & convergence order \\
\hline \hline 10 & 0.073199 & - \\
\hline $10 \cdot 4^{1}$ & 0.019332 & 0.960 \\
\hline $10 \cdot 4^{2}$ & 0.004884 & 0.992 \\
\hline $10 \cdot 4^{3}$ & 0.001224 & 0.998 \\
\hline $10 \cdot 4^{4}$ & 0.000306 & 1.000 \\
\hline $10 \cdot 4^{5}$ & 0.000076 & 1.000 \\
\hline $10 \cdot 4^{6}$ & 0.000019 & 1.000 \\
\hline $10 \cdot 4^{7}$ & 0.000004 & 1.000 \\
\hline
\end{tabular}

Table 1 Results of the HMBO algorithm (15) with temporal discretization only.

\subsection{Convergence analysis under spacial discretization}

Our next tests concern the algorithm's convergence under finite differencing. A circle of radius 0.35 is centered at $(x, y)=(1 / 2,1 / 2)$ with zero initial velocity, and the initial condition for our algorithm is therefore the signed distance function (taking positive values inside the circle) to this interface. The calculations are performed in the unit square $\Omega$ under zero Neumann boundary conditions and we use grid points on a square lattice with spacing $\Delta x=\Delta y=1 /\left(N_{p}-1\right)$, where $N_{p}$ is the number of grid points on the $x$ and $y$ axes. A Delaunay triangulation of these points is performed, and we assume a linear interpolation of the grid values within each element. This allows us to define and determine the precise location of the interface. In particular, the location of the interface at each time step is given as a collection of line segments, each of which corresponds to the zero level set of the signed distance function across the elements. We remark that the geometry of the interface depends on the triangulation and that, as the interface shrinks, the relative resolution of the grid is a source of error. On coarse grids, we observe that this leads to interfacial motions which vanish faster than the exact solution. Since any mesh dependence also leads to a deformation from the exact solution, these changes in shape move the centre of the interface. For this reason we use the centre of the numerical solution in the error computations.

We take the HMBO time step as $\tau=t_{e} / 2^{9}\left(t_{e} \approx 0.4387\right)$ and the time step for the finite difference approximation of the solution to the wave equation is $h=\tau /\left(2^{6}\right)$. We then compute as follows: 
1. Construct the signed distance function $d_{0}(x, y)$ to the initial circle.

2. Set $d_{1}(x, y)=d_{0}(x, y)$ (zero initial velocity), and repeat steps $(3-5)$, for $n=1, \ldots, t_{e} / \tau$.

3. Compute the finite difference solution to the following problem:

$$
\left\{\begin{array}{lr}
u_{t t}=2 \Delta u & \text { in }(0, \tau] \times \Omega \\
\frac{\partial u}{\partial \nu}=0 & \text { on }(0, \tau] \times \partial \Omega \\
u(t=0, x)=2 d_{n}-d_{n-1}, & u_{t}(t=0, x)=0 \quad \text { in } \Omega .
\end{array}\right.
$$

4. Compute $\bar{r}_{n+1}$, the average distance of the data points from their center of gravity. These points describe the zero level set of the solution to (16).

5. Set $d_{n+1}(x, y)=\sqrt{(1 / 2-x)^{2}+(1 / 2-y)^{2}}-\bar{r}_{n+1}$.

The errors are shown in Table 2. Here, $L^{2}$-error designates to the square root of the value below,

$$
\tau \sum_{n=1}^{N_{e}}\left(r((n-1) t)-\bar{r}_{n}\right)^{2}
$$

where $r(t)$ denotes the solution to (13), $N_{e}$ is the largest positive integer satisfying $N_{e} \tau \leq t_{e}$, and the value of $\bar{r}_{n}$ is zero for any iteration after which the approximate solution's radius becomes zero.

\begin{tabular}{|c|c|c|}
\hline Grid Resolution $N_{p}$ & $L^{2}$-error & convergence order \\
\hline \hline 16 & 0.1038 & - \\
\hline 32 & 0.0999 & 0.054 \\
\hline 64 & 0.0929 & 0.105 \\
\hline 128 & 0.0785 & 0.243 \\
\hline 256 & 0.0545 & 0.527 \\
\hline 512 & 0.0283 & 0.946 \\
\hline 1024 & 0.0088 & 1.685 \\
\hline
\end{tabular}

Table 2 HMBO errors with space discretization by FDM (using ideal distance functions).

As our final error analysis, we replace steps 4 and 5 of the previous algorithm with the construction of the signed distance function according to the exact configuration of the piecewise linear approximation of the interface. We again perform the numerical computations on various grid resolutions and investigate the corresponding convergence orders.

The results are show in Table 3 , where we use the conventions of the previous test, and parameter values are the same. We note that explicit computation of the distance functions does not significantly change the order of convergence. 


\begin{tabular}{|c|c|c|}
\hline Grid Resolution $N_{p}$ & $L^{2}$-error & convergence order \\
\hline \hline 16 & 0.1033 & - \\
\hline 32 & 0.0983 & 0.072 \\
\hline 64 & 0.0900 & 0.126 \\
\hline 128 & 0.0739 & 0.286 \\
\hline 256 & 0.0325 & 1.185 \\
\hline 512 & 0.0069 & 2.235 \\
\hline 1024 & 0.0029 & 1.251 \\
\hline
\end{tabular}

Table 3 HMBO errors with space discretization by FDM.

\subsection{Numerical investigations}

The purpose of this section is to computationally investigate properties of the $\mathrm{HMCF}$, including volume preserving and multiphase motions. Our computations make use of the method of minimizing movements, which we employ for the purpose of inquiring into the volume preserving motions.

Since the solution to the wave equation depends only on local information, one can formulate a multiphase algorithm which is akin to the original multiphase MBO algorithm in [13]. In particular, thresholding a collection of independently evolving wave equations can formally be shown to compute multiphase interfacial motions with normal acceleration equal to curvature. Nevertheless, since we would also like to investigate multiphase volume preserving motions, we introduce a reformulation of this idea. In particular, by choosing a small time step $\tau$, we find a function $\boldsymbol{u}: \Omega \rightarrow \mathbf{R}^{K-1}$ solving the following vector valued wave equation:

$$
\left\{\begin{array}{lr}
\boldsymbol{u}_{t t}=\Delta \boldsymbol{u} & \text { in }(0, \tau] \times \Omega, \\
\frac{\partial \boldsymbol{u}}{\partial \nu}=\mathbf{0} & \text { on }(0, \tau] \times \partial \Omega, \\
\boldsymbol{u}_{t}(0, x)=\mathbf{0} & \text { in } \Omega, \\
\boldsymbol{u}(t=0, x)=2 \boldsymbol{z}_{0}^{\epsilon}-\boldsymbol{z}_{-\tau}^{\epsilon} & \text { in } \Omega,
\end{array}\right.
$$

where $K$ denotes the number of phases, $\Omega$ is a smooth bounded domain in $\mathbf{R}^{d}$, and the initial condition is defined using the following signed-distance interpolated vector field (see [5] and [14])

$$
\boldsymbol{z}_{t}^{\epsilon}(x)=\sum_{i=1}^{K}\left(\boldsymbol{p}_{i} \chi_{\left\{d_{i}^{t}(x) \geq \epsilon / 2\right\}}+\frac{1}{\epsilon}\left(\frac{\epsilon}{2}+d_{i}^{t}(x)\right) \boldsymbol{p}_{i} \chi_{\left\{-\epsilon / 2<d_{i}^{t}(x)<\epsilon / 2\right\}}\right) .
$$

Here, $\chi_{E}$ is the characteristic function of a set $E, \epsilon>0$ is the interpolation parameter, the vector $\boldsymbol{p}_{i}$ is the $i^{\text {th }}$ coordinate vector of a regular simplex in $\mathbf{R}^{K-1}$ for $i=1, \ldots, K$, and $d_{i}^{t}(x)$ denotes the signed distance function to the boundary of phase $i$ at time $t$ :

$$
d_{i}^{t}(x)=\left\{\begin{aligned}
\inf _{y \in \partial P_{i}^{t}}\|x-y\| & \text { if } x \in P_{i}^{t} \\
-\inf _{y \in \partial P_{i}^{t}}\|x-y\| & \text { otherwise }
\end{aligned}\right.
$$


At time zero, $\boldsymbol{z}_{-\tau}^{\epsilon}(x)$ is constructed by using (18) on an interface which is created by using the initial velocity to evolve the initial interface backwards in time. We remark that, when $K=2$, equation (17) is scalar.

At time $\tau$, in a process called thresholding, each phase region $P_{i}^{\tau}$ is evolved as follows:

$$
P_{i}^{\tau}=\left\{x \in \Omega: \boldsymbol{u}(\tau, x) \cdot \boldsymbol{p}_{i} \geq \boldsymbol{u}(\tau, x) \cdot \boldsymbol{p}_{k}, \text { for all } k \in\{1, \ldots, K\}\right\} .
$$

The vector field $\boldsymbol{z}_{0}^{\epsilon}$ is then reconstructed using the boundaries of the sets (20) and the initial condition for the wave equation is updated. The procedure is then repeated.

The defining characteristic of the vector field (18) is that, upon choosing a location $x$ positioned within a distance $\epsilon / 2$ of an interface and away from a junction, say corresponding to an interior point of phase $i$ near phase $j$, the vector field satisfies the property:

$$
\left\langle\boldsymbol{z}_{t}^{\epsilon}(x), \boldsymbol{p}_{i}\right\rangle=\frac{K d_{i}^{t}(x)}{\epsilon(K-1)}+\frac{K-2}{2(K-1)},
$$

where the brackets denote the standard Euclidean inner product. From this it follows that the profile in the direction normal to the interface is given by a signed distance function. This fact allows one to obtain the curvature of the interface by computing the Laplacian of (21). Moreover, as shown in [16], this setting introduces a multiple well potential which allows one to treat multiphase volume constrained motions.

Our approximation method for computing multiphase interfacial dynamics thus repeats the PDE step (17) and the thresholding step (20).

\subsection{Minimizing movement approach}

As in the parabolic case [16], the method of minimizing movements can be used to inspect volume constrained interfacial motions. In particular, the minimization formulation of our algorithm enables one to formally include constraints, via penalization. Denoting the prescribed volume of the $i^{\text {th }}$ phase region by $A_{i}$, wave-type minimizing movements can be used under the following functional minimization (see [4]):

$$
\mathcal{F}_{n}(\boldsymbol{u})=\int_{\Omega}\left(\frac{\left|\boldsymbol{u}-2 \boldsymbol{u}_{n-1}+\boldsymbol{u}_{n-2}\right|^{2}}{2 h^{2}}+\frac{|\nabla \boldsymbol{u}|^{2}}{2}\right) d x+\frac{1}{\rho} \sum_{i=1}^{K}\left|A_{i}-\operatorname{meas}\left(P_{i}^{\boldsymbol{u}}\right)\right|^{2} .
$$

Here $\boldsymbol{u}_{n}$ denotes the minimizer of $\mathcal{F}_{n}$ (where $\boldsymbol{u}_{0}$ and $\boldsymbol{u}_{-1}$ are created from the wave equation's initial conditions), $h>0$ is the time step, $\rho>0$ is a small penalty parameter and the areas corresponding to phase regions within $\boldsymbol{u}$ are obtained from the sets:

$$
P_{i}^{\boldsymbol{u}}=\left\{x \in \Omega ; \boldsymbol{u}(x) \cdot \boldsymbol{p}_{i} \geq \boldsymbol{u}(x) \cdot \boldsymbol{p}_{j} \quad \forall j\right\}
$$


Functional values are approximated by means of the finite element method with Lagrange $P 1$ elements, where Delaunay triangulations are used to generate the mesh.

\subsubsection{Unconstrained interfacial motions}

Using the method of minimizing movements to approximate the solution of (17), Fig. 4 shows the result for the evolution of a two phase HMCF, with zero initial velocity. Using a square lattice with node spacing $\Delta x=\Delta y=$ $1 / 99$, parameters were taken as follows: $h=\Delta x / 80, \tau=30 h$. In contrast to parabolic mean curvature flow, we observe oscillations in the interfacial motion and the phase eventually splits apart into two disjoint regions. We note that such topological changes are natural in the hyperbolic setting since one can prescribe the initial configuration, as well as the initial velocity of the interface. To illustrate a multiphase motion, Fig. 5 shows the evolution of a junction
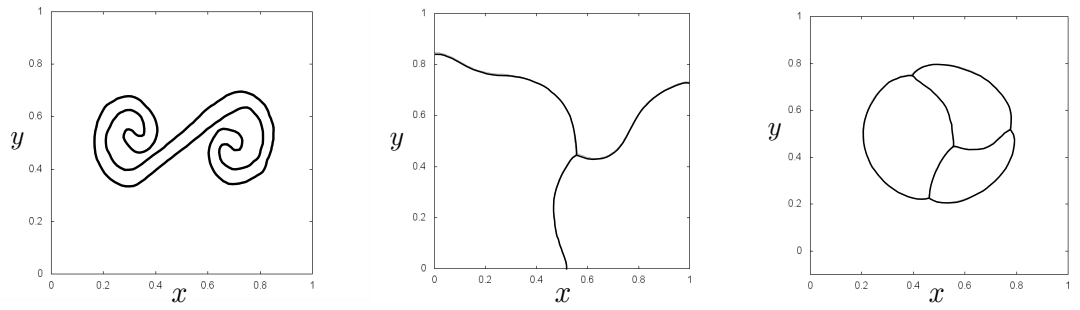

Fig. 3 Initial conditions used in the computations.

obtained by our approximation method (also without the penalty term). We again observe oscillation in each of the interfaces, as well as in the motion of the junction. In this computation, a square lattice of nodes with $\Delta x=\Delta y=1 / 99$ was used to partition the unit square. We took $h=10^{-4}, \tau=40 h$, and the interpolation parameter was $\epsilon=0.07$.

\subsubsection{Volume-constrained interfacial motions}

Fig. 6 shows the two phase volume-preserving motion corresponding to the computations in Fig. 4. In contrast to the standard (parabolic) volume-preserving mean curvature flow, whose evolution approaches a circle, the interfacial dynamics considered here oscillate. Using the same grid as in the previous computations, the parameters were taken as follows: $h=\Delta x / 80, \tau=30 h$, and $\rho=10^{-4}$

Using the multiphase formulation of our algorithm, Fig. 7 shows a four phase oscillatory motion. Computations were performed within the unit square using the same triangulation as was used to produce Fig. 5. The other parameters were $h=10^{-4}, \tau=40 h, \epsilon=0.07$ and we again use a penalty parameter of 


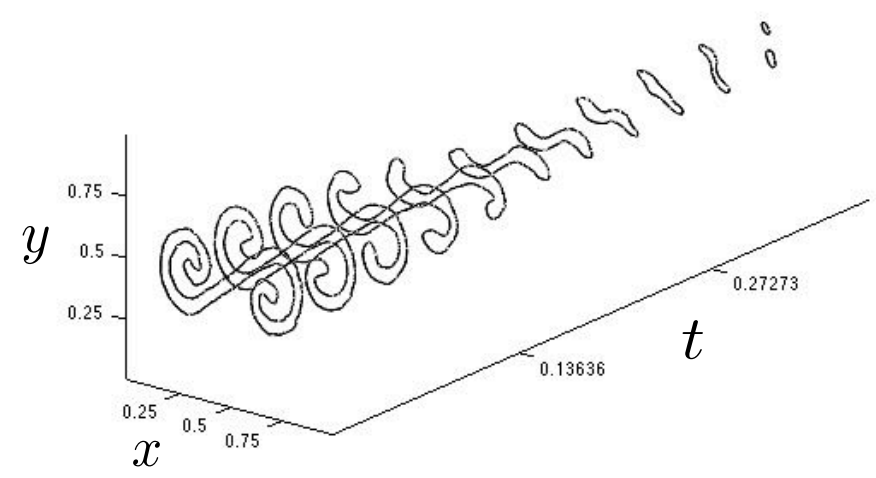

Fig. 4 Evolution by $\boldsymbol{u}^{\prime}=-\kappa \boldsymbol{n}$ (the initial condition is shown at the left of Fig. 3 ).

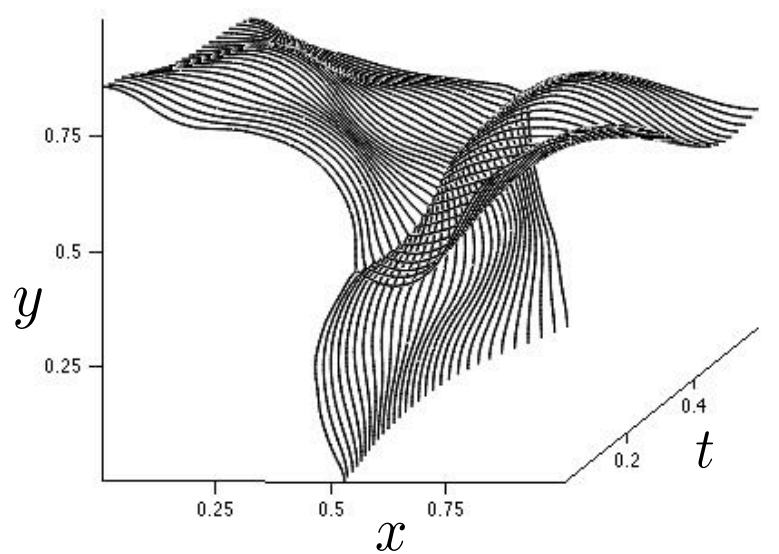

Fig. 5 Motion of a junction under HMCF (the initial condition is shown at the center of Fig. 3).

$\rho=10^{-4}$. In both computations, volumes were preserved to within an absolute error of order $10^{-3}$. 


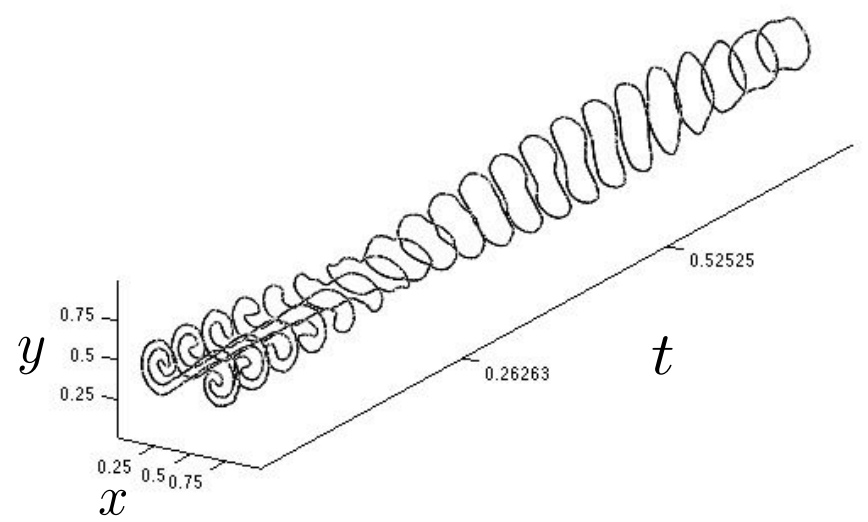

Fig. 6 Volume preserving HMCF (initial condition is shown at the left of Fig. 3).

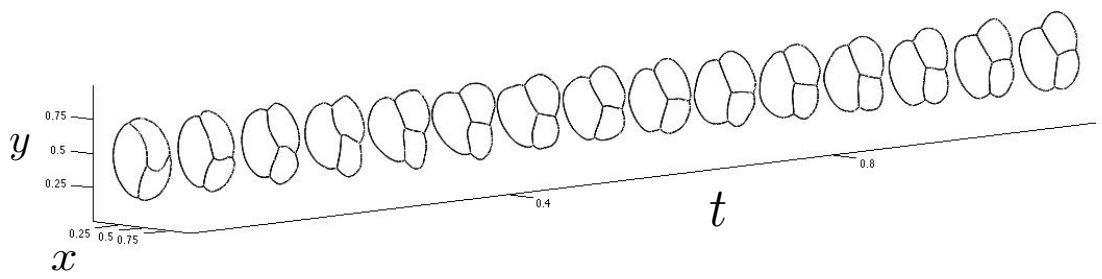

Fig. 7 Multiphase volume preserving HMCF (initial condition is shown at the right of Fig.3).

\section{Conclusion}

We introduced and formally justified a method for approximating interfacial motion governed by curvature dependent acceleration. Our method is a thresholding algorithm of the MBO-type which, instead of utilizing a diffusion process, thresholds an evolution by the wave equation. By means of a combination of signed distance functions in the initial condition for the wave equation, we obtained the desired interfacial dynamics without having to explicitly calculate the interfacial velocity.

We believe that our report opens several interesting directions of research regarding oscillatory interfacial motions. Especially, we would like to investigate numerical methods capable of analyzing the development and propagation of singularities particular to hyperbolic problems. Moreover, the precise formulation, analysis, and numerical solution of the hyperbolic multiphase problem including junctions is a new challenging topic which we want to address in the near future. 


\section{Acknowledgements}

This work was supported by JSPS Grant numbers 25800087, 26870224, and by JSPS A3 Foresight Program "Computational Applied Mathematics 20142019."

\section{References}

1. M. Bonafini, A BMO-type scheme for the relativistic hyperbolic mean curvature flow. Master thesis, Verona University, 2015.

2. S. Essedoglu, S. Ruuth, R. Tsai, Diffusion generated motion using signed distance functions, Journal of Computational Physics 229(4), pp. 1017-1042, 2010.

3. E. Ginder, K. Švadlenka, A variational approach to a constrained hyperbolic free boundary problem, Nonlinear Analysis 71, pp. 1527-1537, 2009.

4. E. Ginder, K. Švadlenka, The discrete Morse flow for volume-controlled membrane motions. Advances in Mathematical Sciences and Applications, 22(1), pp. 205-223, 2012.

5. E. Ginder, A variational approach to volume-controlled evolutionary equations. Dissertation, Kanazawa University, 2012.

6. M. E. Gurtin, P. Podio-Guidugli, A hyperbolic theory for the evolution of plane curves, SIAM J. Math. Anal. 22, pp. 575-586, 1991.

7. C. He, D. Kong, K. Liu, Hyperbolic mean curvature flow, J. Differ. Equs. 246, pp. 473-390, 2009.

8. M. Kang, A level set approach for the motion of soap bubbles with curvature dependent velocity or acceleration. Dissertation, University of California Los Angeles, 1996.

9. K. Kikuchi, S. Omata, A free boundary problem for a one dimensional hyperbolic equation, Advances in Mathematical Sciences and Applications 9, pp. 775-786, 1999.

10. D. Kong, K. Liu, Z. Wang, Hyperbolic mean curvature flow: Evolution of plane curves, Acta Mathematica Scientia 29B(3), pp. 493-514, 2009.

11. D. Kong, Z. Wang, Formation of singularities in the motion of plane curves under hyperbolic mean curvature flow, J. Differential Equations 247, pp. 1694-1719, 2009.

12. P. G. LeFLoch, K. Smoczyk, The hyperbolic mean curvature flow, J. Math. Pures Appl. 90, pp. 591-614, 2008.

13. B. Merriman, J. K. Bence, S. J. Osher, Motion of multiple junctions: A level set approach, J. Comp. Phys. 112, pp. 334-363, 1994.

14. R.Z. Mohammad, K. Švadlenka, Multiphase volume-preserving interface motions via localized signed distance vector scheme, Discrete and Continuous Dynamical Systems Series S 8(5), pp. 969-988, 2015.

15. H. G. Rotstein, S. Brandon, A. Novick-Cohen, Hyperbolic flow by mean curvature, Journal of Crystal Growth 198/199, pp. 1256-1261, 1999.

16. K. Švadlenka, E. Ginder, S. Omata, A variational method for multiphase volumepreserving interface motions, Journal of Computational and Applied Mathematics 257, pp. 157-179, 2014.

17. K. Švadlenka, S. Omata, Mathematical modelling of surface vibration with volume constraint and its analysis, Nonlinear Analysis 69, pp. 3202-3212, 2008.

18. A. Tachikawa, A variational approach to constructing weak solutions of semilinear hyperbolic systems, Advances in Mathematical Sciences and Applications 4, pp. 93-103, 1994. 